CAHIERS DE

NARRATOLOGIE

\section{Cahiers de Narratologie}

Analyse et théorie narratives

32 | 2017

Récit et argumentation, interactions, lieux et dispositifs sociaux

\title{
Vérité et récit dans Le Mépris de Jean-Luc Godard
}

\section{Elsa Grasso}

\section{(2) OpenEdition \\ Journals}

Electronic version

URL: http://journals.openedition.org/narratologie/7811

DOI: 10.4000/narratologie. 7811

ISSN: 1765-307X

Publisher

LIRCES

\section{Electronic reference}

Elsa Grasso, « Vérité et récit dans Le Mépris de Jean-Luc Godard », Cahiers de Narratologie [Online], 32 | 2017, Online since 21 December 2017, connection on 19 April 2019. URL : http:// journals.openedition.org/narratologie/7811; DOI : 10.4000/narratologie.7811

This text was automatically generated on 19 April 2019

Article L.111-1 du Code de la propriété intellectuelle. 


\title{
Vérité et récit dans Le Mépris de Jean-Luc Godard
}

\author{
Elsa Grasso
}

Godard a caractérisé Le Mépris comme son "seul film "classique" "․․ C'est un film qui raconte des histoires archétypales, des histoires comme chacun les aime. Le Mépris est d'abord une histoire d'amour tragique. C'est aussi l'histoire d'un film tourné dans le film, d'un film comme on aimerait en réaliser: digne d'illustrer avec nostalgie la grande époque hollywoodienne, avec un réalisateur mythique (Lang), une lourde caméra américaine, la Mitchell, sur rails, manœuvrable par de multiples jeux de manivelles ; avec les fameux studios de Cinecittà, et la splendeur de Capri. Enfin, l'histoire que raconte ce film dans le film est un sujet immémorial, sans doute le récit d'aventure le plus célèbre qui soit : celui, épique, des détours sur la mer d'un homme valeureux qui aime sa femme, son fils et son pays, et qui veut rentrer chez lui. On sait que Godard reprend au début du film la formule d'André Bazin selon laquelle «le cinéma substitue à notre regard un monde qui s'accorde à nos désirs »; il ajoute : «Le Mépris est l'histoire de ce monde ». Faire le récit de ce monde consiste ainsi pour ce film à raconter, dans le même mouvement, une histoire tragique et celle de la transformation en récit cinématographique du texte poétique. Et cela revient par là même à raconter l'histoire d'un retour, celui d'Ulysse, mais aussi un retour au monde, et un retour à ce que nous attendons de trouver au fond de tout grand récit.

2 Il s'agira ici, pour approcher ce récit nostalgique, d'analyser la relation que peut entretenir la dimension narrative, ainsi triplement actualisée, avec la modalité de la simplicité, d'une part, comme mesure et valeur de la représentation, et avec la question de la vérité, d'autre part, comme visée persistante d'une représentation pourtant non soumise au modèle de l'adéquation ni à celui, plus généralement, de la valeur épistémique. 


\section{Diffractions du récit}

3 L'adaptation du "roman de gare » de Moravia (comme Godard désignait lui-même l'ouvrage) est une magistrale spéculation sur l'adaptation cinématographique, et tout autant l'un des plus éblouissants hommages contemporains au récit odysséen. Le Mépris semble bien nous mettre sous les yeux l'essentiel, dans une éclatante et définitive trichromie. Avant tout, le film montre. Il montre les passions (Camille et Paul) ; le destin (les dieux, les accidents de voiture) ; la puissance des récits et des images (la caméra braquée, au début, sur nous; ou, à la fin, sur Ulysse vu de dos, regardant lui-même Ithaque retrouvée). Le Mépris semble donc représenter (par une mimêsis et une diêgêsis), sans rien démontrer.

4 Selon une perspective tout aristotélicienne, la connaissance se déploie, par la diégèse et la mimèsis, comme reconnaissance. L'œuvre la plus populaire de Godard semble bien, en effet, nous faire connaître ce que nous connaissons le mieux, et que nous retrouvons là, dans son essence rendue à nous transparente, sous l'aspect de sa mimêsis racontée et filmée. L'objet de narration n'y est rien d'autre que l'essentiel de l'humaine condition, de la violence du destin, et de la nécessité non pas seulement de le vivre, mais aussi de le représenter, par le verbe poétique ou par l'image cinématographique. Il s'agit bien d'un film-monument, et cela s'accompagne toujours d'une forme d'évidente et triomphante simplicité :

Film simple et sans mystère, film aristotélicien, débarrassé des apparences, Le

Mépris prouve, en 149 plans [176 après montage] que dans le cinéma comme dans la

vie, il n'y a rien de secret, rien à élucider, il n'y a qu'à vivre - et à filmer².

Il y a une dimension d'heureuse redécouverte propre à ce récit cinématographique, dont la simplicité tient en partie à ceci qu'il raconte seulement ce que chacun connaît le plus intimement (la souffrance amoureuse, l'émotion provoquée par la splendeur du monde, la nécessité pour les humains de dire le monde). Pourquoi Godard, dans ce commentaire, recherche-t-il chez Aristote une modalité esthétique adéquate? Peut-être parce que l'auteur de la Poétique théorise une mimêsis positivement évaluée, facteur de connaissance et non pas d'illusion. Le Mépris emprunterait sans doute sur ce point à des schèmes et des présupposés esthétiques nettement plus aristotéliciens que platoniciens.

Cependant, cette puissance heuristique du récit s'accompagne aussi d'une forme de diffraction, d'une résistance qui semble défaire la narration et la priver de toute vertu épistémique. Cette diffraction et cette résistance opèrent constamment dans la lenteur erratique du plan séquence, dans la régularité hypnotique, au générique, du lent travelling initial de la caméra sur rails, des ellipses attachées aux mouvements des personnages ou des ellipses de leurs trajets ${ }^{3}$, ou enfin dans la quasi immobilité silencieuse des plans fixes sur la Méditerranée.

7 Quelle forme de narration opère donc dans ce qu'Aragon a pu considérer comme une œuvre proprement géniale ${ }^{4}$ ? Qu'est-ce qui peut bien venir, ici, s'articuler à cette " simplicité » revendiquée par Godard lui-même ? Comment, aux caractères « classiques » d'une narrativité épistémiquement structurée, peuvent bien participer des dimensions intrinsèques beaucoup plus erratiques, voire a-logiques ? La qualification, par Godard, de son film comme d'un « film simple » peut ainsi surprendre, lorsqu'on prend précisément en compte ses dimensions fort peu classiques et plutôt complexes. 
8 La puissance singulière du film prend sa source dans un aspect qui pourrait sembler, superficiellement, très conventionnel: son caractère romanesque. Deleuze considère Godard comme le plus « romanesque » de la Nouvelle vague.

Le cinéma cesse d'être narratif, mais c'est avec Godard qu'il devient le plus « romanesque ». (...) Bakhtine définissait le roman, par opposition à l'épopée ou à la tragédie, comme n'ayant plus l'unité collective ou distributive par laquelle les personnages parlaient encore un seul et même langage. Au contraire, le roman emprunte nécessairement tantôt la langue courante anonyme, tantôt la langue d'une classe, d'un groupe, d'une profession, tantôt la langue propre d'un personnage. Si bien que les personnages, les classes, les genres, forment le discours indirect libre de l'auteur, autant que l'auteur forme leur vision indirecte libre (ce qu'ils voient, ce qu'ils savent ou ne savent pas). (...). Bref, c'est la réflexion dans les genres, anonymes ou personnifiés, qui constitue le roman son "plurilinguisme», son discours et sa vision. Godard donne au cinéma les puissances propres du roman ${ }^{5}$

De là naît une « ligne brisée, une ligne en zigzag, qui réunit l'auteur, ses personnages et le monde, et qui passe entre eux ». Et Godard constitue ainsi un exemple frappant, pour Deleuze, du "cinéma moderne», en tant qu'il met en évidence « de nouveaux rapports avec la pensée $»^{6}$.

Les dimensions a-logiques du Mépris pourraient être saisies, selon l'approche de Deleuze, comme "relations non localisables", propres à une "narration cristalline"; ou encore, sur le plan de la description, comme participant à des «situations optiques et sonores pures, devenus voyants, ne peuvent plus ou ne veulent plus réagir, tant il faut qu'ils arrivent à "voir" ce qu'il y a dans la situation $»^{7}$. Dans la Nouvelle vague précise Deleuze, « (...) la vision (...) prend toute la place et tient lieu d'action. »

11 Il y a, dans Le Mépris, une énigme et une évidence enchâssées l'une dans l'autre, qui sont celles du parallélisme, de l'indépendance réciproque et, en même temps, de la corrélation inexplicable de deux registres, celui des dieux - ou du destin - et celui des actions et des passions humaines.

De ce point de vue, naturellement, le film de Godard répète avec une certaine fidélité un motif antique : celui du destin tragique qui accable les hommes (« Il faut souffrir», dit Lang à Paul Javal-Michel Piccoli, repoussé par sa femme Camille), et qui se déroule toujours inexorablement sous le regard divin ; celui-ci surplombe, sans le partager, le sort des humains. De même le regard des statues de dieux, dans le film, n'interfère pas dans la sphère narrative et croise comme de nulle part le regard du spectateur. Une autre modalité de ces différences de strates ou de registres est donnée aussi lorsque ces regards de dieux, descendant du haut d'un ciel olympien, deviennent pour un instant notre propre point de vue de spectateur (par la vertu d'une caméra hissée en hauteur), lors de la scène où nous voyons comme d'en haut Camille puis Paul : traversant l'espace du toit terrasse de la Villa Malaparte comme ils le feraient d'une scène de théâtre tragique, terrible scène grandeur nature, encerclée des rochers gigantesques plongeant dans l'azur. C'est le monde lui-même, ici, qui semble devenu un spectacle tragique sous des regards divins inexorables.

13 À cette première modalité de diffraction vient répondre la fracture entre les images du film de Lang (Lang personnage de Godard), et les scènes narrant l'histoire de Paul et de Camille. Fracture, et pas seulement séparation, car ce sont deux mondes inconciliables, qui se déploient pourtant de façon détonante dans le même cadre et comme juxtaposés l'un à l'autre, alors même qu'ils ne peuvent en aucun cas coexister dans le même espace- 
temps. Les images de Lang, loin d'être insérées dans le film comme des images de fiction dotées d'un degré de réalité inférieur ou moins véritable, apparaissent au contraire comme déployant un monde tout aussi entier et tout aussi existant que celui dans lequel se débattent Paul et Camille. Certes, le maquillage et les costumes des personnages grecs sont outrés et artificiels, mais le monde qui est censé être celui qu'habitent ces personnages apparaît, lui, comme bien réel, et en même temps sous un jour nouveau, dans les images de Lang : le personnage interprétant Ulysse, ou les sirènes, nageant dans les eaux de Capri, font surgir leur monde propre, nouveau (et ancien), celui de la Méditerranée de la poésie grecque archaïque, qui n'est plus le même que celui où interagissent les personnages modernes.

(...) la situation géographique de la villa (Malaparte) est importante. Seule face à la mer, elle renforcera l'idée d'un monde odysséen, en lui donnant une réalité et une présence quasi palpable.

(...) Dans tout le film, le décor ne doit être utilisé que pour faire sentir la présence

d'un autre monde que le monde moderne de Camille, Paul et Jérémie Prokosch ${ }^{8}$.

Les deux catégories représentatives, inconciliables, se déploient pourtant selon une contemporanéité et une équivalence qui, pour être impossibles, n'en sont pas moins effectives. Nous voyons donc : a/ la construction d'une fiction cinématographique (le film de Lang) représentée au sein du récit cinématographique (le film de Godard), et $b /$ la puissance d'apparition du monde de l'odyssée, comme objet poétique, représenté par ladite fiction. Mais il en va de même, d'autre part, pour la relation entre $: c /$ le registre du récit épique, et $\mathrm{d} /$ celui de la scène conjugale. Et la puissance du récit, dans le Mépris, se tient précisément dans le caractère, irrationnel et cependant manifeste, de cette équivalence entre les deux registres. Il s'agit d'une équivalence tant de la puissance et de la dignité représentatives que de la signification: les spectateurs se trouvent également sensibles à ces deux mondes, et à ces deux registres, l'un épique et poétique, l'autre trivial et tragique, sans pouvoir hiérarchiser le niveau de réalité et de fiabilité des deux contenus de représentation et des deux catégories représentatives y opérant ${ }^{9}$.

La distance entre les deux registres est de ce fait annulée en même temps qu'elle continue d'être latente. Cette dimension contradictoire d'une distance irréductible assortie d'une contiguïté immédiate opère donc aussi bien entre le monde des dieux et celui des hommes.

Nous pouvons dans cette perspective renvoyer à la dimension principielle, largement opératoire dans le film, de la nostalgie. Celle-ci est évidemment à l'œuvre dans toutes les scènes représentant l'Odyssée, ce poème du mal du retour, nostos. Mais elle est aussi directement déployée, on le sait bien, dans le renvoi à un âge d'or du cinéma, ainsi que dans le mouvement de retour vers un monde antique grec et romain. Or la nostalgie, en s'exerçant dans les deux registres, projette le monde lui-même, ainsi que la réalisation cinématographique, dans une sorte de retrait ou de distance infinitésimale, qui produit l'impression que les objets désirés sont là, tout proches, mais cependant inatteignables complètement dans la représentation. On ne peut dire, quand on voit Le Mépris, lequel, du monde odysséen ou de celui des personnages modernes, nous est le plus immédiatement donné; lequel est représenté comme plus réel que l'autre; ni lequel est le plus directement posé comme objet de nostalgie.

17 Cette égale et contradictoire actualisation de registres représentatifs irréconciliables produit l'impression que (a) le monde est à la fois pleinement donné par le récit, surgissant hors de celui-ci comme les rochers hors des flots bleus de Capri; et que (b) le 
même monde est en même temps distancié : toujours et inlassablement visé, et cependant toujours mis en retrait (au lieu d'être pleinement déployé à l'intérieur du récit, comme c'est le cas pour une narrativité cinématographique académique, et, pour reprendre le mot de Godard, «hollywoodienne »). C'est bien le sentiment «moderne » que Godard associe à l'esprit de son film :

Je vois tout à fait Le Mépris comme s'il ne restait plus personne sur la terre. Il y a cinq rescapés. Voilà ce qu'ils font. Le cinéma remplace le regard des dieux. Il se rapproche. Les dieux n'ont jamais fait autre chose que se rapprocher des hommes. (...) Le Mépris est l'histoire des hommes qui se sont coupés d'eux-mêmes, du monde, de la réalité. Ils essaient maladroitement de retrouver la lumière, alors qu'ils sont enfermés dans une pièce noire ${ }^{10}$.

L'Odyssée est le récit d'un homme qui souffre du mal du retour, et les personnages de Godard qui doivent représenter ce récit homérique au cinéma souffrent eux aussi d'un retour impossible. Ce retour nous ferait revenir à une relation immédiate au monde d'Homère et à sa réalité vécue. Celui-ci nous apparaît encore; mais ce monde, pour être réel, ne nous en est pas moins retiré. C'est ce que décrit Lang (personnage de Godard, plan 139) :

Le monde d'Homère est le «monde réel appartenant à une civilisation qui s'est développée en accord et non en opposition avec la nature, et la beauté de l'Odyssée réside justement en cette croyance en la réalité comme elle est.

19 Aussi Deleuze peut-il écrire : «Le cinéma cesse d'être narratif, mais c'est avec Godard qu'il devient le plus "romanesque" ». Deleuze renvoie ici à la pluralité des points de vue sur un même objet de narration, qu'il assigne en propre au roman. Dans le cas du Mépris, il s'agira de la fin de la relation amoureuse de Paul et Camille, qui est représentée du point de vue de Camille, de celui de Paul, mais aussi de celui d'une variété de personnages extrêmement différents (Lang, Francesca, Prokosch).

Et Deleuze poursuit, au sujet de Godard, retrouvant remarquablement la description par Godard lui-même du rapport au monde qu'il a voulu représenter dans Le Mépris :

(...) le cinéma moderne développe ainsi de nouveaux rapports avec la pensée :

l'effacement d'un tout ou d'une totalisation des images, au profit d'un dehors qui s'insère entre elles (...), l'effacement de l'unité de l'homme et du monde, au profit d'une rupture qui ne nous laisse plus qu'une croyance en ce monde-ci ${ }^{11}$.

\section{"Le Mépris est un film simple et sans mystère, film aristotélicien, débarrassé des apparences. »}

Reprenons pas à pas cette formule de Godard, citée plus haut.

A/ «Le Mépris est un film simple » : un film élémentaire.

«Un film simple» signifie d'abord un film non complexe: il ne représente que de l'élémentaire. Grâce à Descartes, nous savons qu'il est épistémologiquement nécessaire et bénéfique, pour la représentation, de procéder du simple vers le composé, de partir du connu. Dans le cas du film de Godard, nous partons vraiment de l'élémentaire ; la bande annonce de 1963 égrène des concepts premiers de la narration, du mythe, de la fiction universelle, du cinéma : la Femme, l'Homme, la trahison, la mort... Mais cela nous mènera à une forme de simplicité encore plus pure, non au complexe. Les personnages avancent pas à pas, vers le tragique (la mort) et vers le retrait du discours : le dernier mot du Mépris , lorsque la caméra filme la mer étale éblouissante, le mot de la fin donc, sera «Silenzio ». 
24 - Le Mépris, adaptant un roman de gare, est une très banale histoire d'amour, qui répète et redit toutes les histoires d'amour au cinéma.

- Il revient aussi au simple en posant les questions principielles: ainsi la nature de l'herméneutique du cinéma. Qu'est-ce que chercher le sens d'une œuvre universelle quand on veut l'adapter au cinéma (pourquoi Ulysse ne rentre-t-il pas chez lui ? c'est un des dialogues Lang-Javal). Et qu'est-ce que transformer en images le texte ou le langage d'un poème? Ici, avec l'Odyssée, il s'agit pour les personnages filmés par Godard (réalisateur, scénariste, producteur) de dire un monde, le monde grec. Comment celui-ci sera-t-il dit ? Par le plus simple : le plus indécomposable. Donc pas le mot ni la forme, ni la structure, mais la matière même de la sensation (visuelle) : la couleur, c'est-à-dire ce que la philosophie classique et moderne (Descartes dans la Dioptrique, mais aussi la logique de Port Royal, et Kant) a toujours opposé à la forme, au dessin, lesquels portent et attestent l'intelligibilité. Et seront privilégiées par Godard les couleurs, précisément, élémentaires ; on sait l'importance dans le film de la trichromie. Dans son scenario, il souligne :

Toute la deuxième partie sera dominée du point de vue couleurs par le bleu profond de la mer, le rouge de la villa et le jaune du soleil, on retrouvera ainsi une certaine trichromie assez proche de celle de la statuaire antique véritable ${ }^{12}$.

Celle-ci est mise en évidence dans la première partie, lors du plan séquence dans l'appartement : jaune, rouge et bleu marqués par les vêtements et les objets. Elle l'est aussi dans la deuxième partie, à Capri, avec le jaune du soleil, de la serviette de Camille, le rouge de la villa de Malaparte et des rochers, le bleu du ciel et de la mer, la peinture sur les statues des dieux grecs.

B/ Le Mépris est " un film simple », au sens aussi où il est "sans mystère ». Il s'agit d'une narration qui n'a rien à élucider, qui n'a aucun secret à déceler ou à révéler.

- L'amour même y est, dès le début, une histoire de surface : une histoire de peau : ce que met sous les yeux la fameuse séquence initiale du corps nu de Bardot. Le désir porte sur la beauté de la surface. Le dialogue amoureux n'a pas à être plus profond. Le superficiel est le tout de l'amour. Il n'y a rien à dire d'autre ; " aimer totalement ", selon l'expression de Camille dans cette scène fameuse, c'est aimer toute la surface d'un corps, ce que l'on en voit.

28 L'aventure amoureuse n'est rien d'autre que ce que l'on voit apparaître: un homme ordinaire qui ne résiste pas vraiment à l'idée d'user de la séduction de sa femme pour un profit social, et le mépris que cela lui vaudra de la part de son épouse. Les deux éléments se voient absolument, il n'y a rien d'autre à chercher dans l'histoire.

29 - Les sentiments, de même, sont parfaitement apparents à la surface: ils se voient pleinement sur le visage, comme sur des masques de théâtre grec aux expressions affichées; on pense bien sûr au gros plan sur le visage de Camille, à Capri après que son mari Paul l'a encouragée à rentrer à la villa avec le producteur. C'est une reprise de la scène initiale de Cinecittà où le même masque tragique apparaissait dans la voiture de Prokosch. Dans la scène définitive à Capri, on se rappelle de Camille la moue de mépris, les lunettes noires qui ne cachent pas de complexité intérieure. Et les commentaires de Godard accompagnent cette présence sans fard: il considère que Camille est entière, absolument lisible, et attend de Bardot qu'elle joue de façon végétale : avec une simplicité de plante, pour laquelle n'existent que des actes tels que la vie, la santé, l'épanouissement sans réflexion : des états élémentaires. Et c'est cette simplicité qui fait la tragédie ${ }^{13}$. 

la mer et son île, les bras levés. de l'existence. mimesis, et cela sans tromperie.

Les sentiments sont donc, eux-mêmes, simples. Ils sont manifestes, et sont non complexes, non composés : un amour entier ; un mépris complet. Sans transition.

1 - Le monde, enfin, est simple : il se montre comme surface, avec l'évidence d'une clarté entière. Le film est envahi par cette autre toile, omniprésente, de la mer; celle-ci est encore soulignée par la massivité brute des rochers s'enfonçant dans l'eau. Il n'y a pas de plan sous-marin; c'est une eau translucide, sans secret, vue depuis la surface et qui ne cache rien, à travers laquelle on voit les corps nageant. Le monde se dit à nouveau comme couleur du ciel et des rochers. Il y a sur ce monde une lumière pleine et sans ombre : rien d'obscur, de demi-caché, rien qui se présente comme à révéler.

- Le cinéma, lui aussi, cette toile que peindra Lang, montre une surface. C'est ce que laisse entendre Paul lors du tournage du film à Capri : «c'est extraordinaire le cinéma ; on voit des femmes, elles ont des robes, le cinéma arrive, on voit... ». Le cinéma raconte en dévoilant les surfaces visibles. Qu'est-ce que réaliser un film? Ce que fait avant tout le personnage de Lang. Il est tout regard : il observe sans cesse, interagit peu.

C/ «Un film débarrassé des apparences ; aristotélicien ».

Paradoxe du Mépris: il ne montre que de la surface, et pourtant il est «débarrassé des apparences ", dit Godard. Car l'apparence implique l'existence de son contraire: la profondeur, l'essence, la réalité cachée car profonde. Avec Le Mépris, il n'y a plus d'apparences parce qu'il n'y a plus que de l'apparaître : éclat en cinémascope de Capri, de la beauté d'un corps nu. Le Mépris raconte un monde qui n'est pas à déchiffrer, mais seulement à voir. Et il nous laisse entendre que les hommes n'y arrivent pas, car ils sont déchirés par leurs sentiments et leurs relations. Par qui alors le monde se donne-t-il à voir? Il n'est vu comme monde que par des sujets hors récits : les dieux. Ceux-ci dans le Mépris sont purs regards; visages de statues surplombant le monde et les humains. Et cette apparition du monde consiste aussi en l'apparition des aventures des hommes: Camille-Paul, ou Ulyssse ; aventures faites de sentiments simples et tragiques. C'est cela que les dieux regardent, que le cinéma nous donne à voir.

Le cinéma de Godard dans le Mépris vient donc coïncider avec le regard des dieux. Quant aux héros, ils seront dès lors ceux qui peuvent regarder presque comme des dieux, ceux qui voient toute la surface du monde : ce que fait Lang, et ce que fait son Ulysse. Dans de la dernière image du film de Lang à Capri, Ulysse est tout regard : se tenant de dos, il fixe

C'est bien là une simplicité radicale : le récit est ce qui fait voir la figure du monde. On raconte toujours, en définitive, dans tout grand récit, ce qui se dit de l'autre côté des aléas

D/ Pourquoi ajouter que c'est là un «film aristotélicien »? Il arrive à Godard de rappeler qu'Aristote est le théoricien du syllogisme. Mais ici, ce qui importe est sans doute essentiellement que la mimèsis est, avec Aristote, lestée de valeur épistémique. Le récit est

Le Mépris n'est pas un film sur l'apparence, si celle-ci implique une vérité ou une essence cachée en dessous d'elle. On doit ici renvoyer aux analyses que Deleuze rassemble sous le titre « les puissances du faux ». (Il emprunte en fait l'expression à Alain Bergala ${ }^{14}$ ).

Deleuze met en avant deux réalisateurs pour illustrer ce qu'il entend par les puissances du faux au cinéma: Wells et Lang. Et il associe Lang au personnage philosophique et platonicien de Protagoras, celui-là même dont Aristote expliquait, comme l'avait fait 
Platon, qu'il n'acceptait pour réalité que des phénomènes dépourvus d'arrière-fond substantiel :

Pour Lang, on dirait qu'il n'y a plus de vérité, mais seulement des apparences. Lang américain devient le plus grand cinéaste des apparences, des fausses images (...). Tout est apparence, et pourtant ce nouvel état transforme le système du jugement plutôt qu'il ne le supprime. En effet, l'apparence est ce qui se trahit soi-même; les grands moments chez Lang sont ceux où un personnage se trahit : Les apparences se trahissent, non pas parce qu'elles feraient place à une vérité plus profonde, mais simplement parce qu'elles se révèlent elles-mêmes comme non-vraies: le personnage fait une gaffe, il connaît le prénom de la victime («L'invraisemblable vérité »), ou bien il sait l'allemand («Les bourreaux meurent aussi»). Dans ces conditions, il reste possible de faire surgir de nouvelles apparences sous le rapport desquelles les premières seront jugeables et jugées (...). Lang invente un relativisme à la Protagoras, où le jugement exprime le point de vue 'le meilleur', c'est-à-dire le rapport sous lequel les apparences ont une chance de se retourner au profit d'un individu ou d'une humanité de plus haute valeur ${ }^{15}$.

En termes deleuziens, Le Mépris privilégie donc l'image-temps, et non pas l'imagemouvement, en raison de sa simplicité même. Il ne raconte pas une aventure, un schème spatio-temporel où se dirait un vrai susceptible de s'opposer à un faux. Il dit le phénomène même du monde comme ce qui est simplicité irréductible, non susceptible de devenir objet d'un jugement séparant le vrai du faux: apparaître de la couleur, de la beauté, de la mort, surface et plénitude qui se rencontrent dans des stases, des états d'immobilité qui parsèment le récit, dans l'histoire de Paul et Camille comme dans l' Odyssée de Lang; dans l'appartement romain, dans la villa de Malaparte, sur le canot de tournage, sur le toit de la villa. Le récit est la mise en scène des états immobiles où se dit l'impossibilité de trouver un secret véritable derrière des apparences, parce qu'il n'y a que de la surface brillante, pleinement réelle.

\section{NOTES}

1. Godard déclare en 1985 : «ça a été le seul film “classique” que j’aie eu l'occasion de faire dans le système ; c'est un film un peu hollywoodien " (Jean-Luc Godard par Jean-Luc Godard. Tome I : 1950-1984, édition établie par Alain Bergala, Cahiers du cinéma, Ed. de l'Etoile ( $1^{\text {re }}$ éd. 1985), 1998, nouvelle éd., p. 16).

2. Cahiers du cinéma, n¹46, Août 1963 ; cité in Jean-Luc Godard par Jean-Luc Godard. Tome I : 1950-1984, op. cit., p. 249.

3. Pensons à la coupure brutale entre la partie du film se déroulant à Rome (plan 139), et l'ouverture de la première scène se déroulant à Capri : visage de Camille apparaissant soudain en gros plan, baigné de la lumière du soleil sur la mer. Et ellipse, par exemple, du trajet de Camille en hors-bord avec Prokosch, avec, en lieu et place, un plan sur un Neptune évoquant le destin (plan 151).

4. Peu après la sortie du film, Aragon, rédacteur en chef des Lettres françaises, écrit un «Hommage à Jean-Luc Godard». Il y souligne l'aspect romanesque du film : "J'en ai vu un, de roman d'aujourd'hui. Au cinéma. Ça s'appelle Le Mépris. Le romancier est un nommé Godard. L'écran français n'a rien eu de mieux depuis Renoir, quand Renoir état le romancier Jean Renoir. 
Je n'arrive pas à comprendre les réserves que j'ai lues, touchant ce film, ailleurs et dan mon propre journal. Tiens, on me demandait du génie, eh bien, le voilà, le génie.» (Les Lettres françaises 6/2/1964).

5. G. Deleuze, Cinéma 2. L'image-temps, Paris, Les Editions de Minuit, 1985, p. 244.

6. Ibid., p. 245 .

7. Ibid, p. 168-169.

8. Extrait du scénario du film, in Jean-Luc Godard par Jean Luc Godard, Tome I, op. cit., p. 241 sq.

9. «Les scènes de l'Odyssée proprement dite, c'est-à-dire les scènes que tourne Fritz Lang en tant que personnage, ne seront pas photographiées de la même façon que celles du film lui-même. Les couleurs en seront plus éclatantes plus violentes, plus vives, plus contrastées, plus sévères aussi, quant à leur organisation. Disons qu'elles feront l'effet d'un tableau de Matisse ou Braque au milieu d'une composition de Fragonard ou d'un plan d'Eisenstein dans un film de Rouch » (ibid.).

10. Entretien avec Jean Collet, in Jean Collet, Jean-Luc Godard, Seghers, coll. Cinéma d'aujourd'hui, Paris, 1963.

11. G. Deleuze, Cinéma 2, op. cit., p. 244-245.

12. Jean-Luc Godard par Jean Luc Godard, Tome I : 1950-1984, édition établie par Alain Bergala, Cahiers du cinéma 1985 (extrait du scénario du film), p. 241 sq.

13. «Camille agit non psychologiquement, si l'on peut dire, par instinct, une sorte d'instinct vital comme une plante qui a besoin d'eau pour continuer à vivre. (...), elle existe sur un plan purement végétal (...). Elle vit de sentiments pleins et simples, et n'imagine pas de pouvoir les analyser. Une fois le mépris pour Paul entré en elle, il n'en sortira pas, car ce mépris (...), n'est pas un sentiment psychologique né de la réflexion, c'est un sentiment physique comme le froid ou la chaleur, rien de plus (...) ; et voilà en fait pourquoi le Mépris est une tragédie. » (extrait du scénario du film, in Jean-Luc Godard par Jean Luc Godard, Tome I, op. cit., p. 241 sq.).

14. Voir la note de Deleuze lui-même, Cinéma 2. L'image-temps, op. cit., chap «Les puissances du faux ", p. 173 (cf. Cahiers du cinéma, n 351, «Le vrai, le faux, le factice »; sept. 1983 ; à propos d'une planche d'images de Welles).

15. G. Deleuze, Cinéma 2. L'image-temps, op. cit., p. 180-181.

\section{ABSTRACTS}

This paper tackles the relationship in Godard's movie Contempt (Le Mépris, 1963) between the many modes of narration and the issue of truth. In particular, an analysis is given of Godard's statement: "Contempt is a simple movie, with no mystery", an "Aristotelian movie, all seeming removed." The issues of simplicity and seeming are analysed in order to show the meaning and nature of truth in Contempt, and also how the film can be understood using Deleuzian concepts of these issues.

Cet article étudie les relations à l'œuvre, dans Le Mépris, entre les multiples modalités du récit et la question de la vérité. On analyse en particulier le sens que peut revêtir la formule de Godard selon laquelle: «Le Mépris est un film simple et sans mystère», un "film aristotélicien, débarrassé des apparences ». La question de la simplicité et celle des apparences sont en particulier analysées de façon à faire apparaître quel sens et quelle consistance revêt, dans ce film, la notion de vérité, et comment Le Mépris est compréhensible au moyen des concepts deleuziens liés à cette question. 
INDEX

Keywords: Mépris, Godard, truth, narration, Deleuze

Mots-clés: Mépris, Godard, vérité, récit, Deleuze

\section{AUTHOR}

\section{ELSA GRASSO}

Université Nice Sophia Antipolis 